\title{
Metagenomic Discovery of CRISPR-Associated Transposons
}

\author{
James R. Rybarski",1, Kuang Hü,1,2, Alexis M. Hill*,2, Claus O. Wilke², Ilya J. Finkelstein ${ }^{1,3}$ \\ ${ }^{1}$ Department of Molecular Biosciences, The University of Texas at Austin, Austin, Texas 78712, USA \\ ${ }^{2}$ Department of Integrative Biology, The University of Texas at Austin, Austin, Texas 78712, USA \\ ${ }^{3}$ Center for Systems and Synthetic Biology, The University of Texas at Austin, Austin, Texas 78712, USA \\ * equal contribution \\ To whom correspondence should be addressed: \\ Claus O. Wilke: wilke@austin.utexas.edu \\ Ily a J. Finkelstein: ilya@finkelsteinlab.org
}

\begin{abstract}
$\underline{\text { Abstract }}$
CRISPR-associated transposons (CASTs) co-opt Cas genes for RNA-guided transposition. CASTs are exceedingly rare in genomic databases; recent surveys have reported Tn7-like transposons that co-opt Type I-F, I-B, and V-K CRISPR effectors. Here, we expand the diversity of reported CAST systems via a bioinformatic search of metagenomic databases. We discover new architectures for all known CASTs, including novel arrangements of the Cascade effectors, new self-targeting modalities, and minimal V-K systems. We also describe new families of CASTs that have co-opted the Type I-C and Type IV CRISPR-Cas sy stems. Our search for non-Tn7 CASTs identifies putative candidates that co-opt Cas 12a for horizontal gene transfer. These new sy stems shed light on how CRISPR systems have co-evolved with transposases and expand the programmable gene editing toolkit.
\end{abstract}

Keywords: gene editing, bioinformatics, CAST, transposition, CRISPR RNA

\section{Introduction}

CRISPR-associated transposons (CASTs) are transposons that have delegated their insertion site selection to a nuclease-deficient CRISPRCas system. All currently-known CASTs derive from Tn7-like transposons and retain the core transposition genes TnsB and $\mathrm{TnsC}$ but dispense with TnsD and TnsE, which mediate target selection $(1,2)$. Tn7 transposons site-specifically insert themselves at a single chromosomal locus (the attachment or att site) via the TnsD/TniQ family of DNAbinding proteins, while TnsE promotes horizontal gene transfer onto mobile genetic elements. In contrast, Class 1 CASTs replace TnsD and TnsE with a crRNA-guided TniQ-Cascade effector complex (3-6). These CASTs can use the TniQ-Cascade complexes for both vertical and horizontal gene transfer (5). One notable exception is a family of Type IB CASTs that retains TnsD for vertical transmission but co-opts TniQCascade for horizontal transmission (7). Similarly, Class 2 CASTs use the Cas $12 \mathrm{k}$ effector to transpose to the attachment (att) sites or to mobile genetic elements $(8,9)$. CASTs also dispense with the spacer acquisition and DNA interference genes found in traditional CRISPR-Cas operons (2). In short, these systems have merged the core transposition activities with crRNA-guided DNA targeting.

CASTs are exceedingly rare; only three sub-families of $\operatorname{Tn} 7-$ associated CASTs have been reported bioinformatically and experimentally $(2,5,7,9,10)$. These studies have identified that many, but not all, CASTs encode a self-targeting spacer flanked by atypical (privileged) direct repeats. However, the prevalence of such atypical repeats, the diversity of self-targeting strategies, and the molecular mechanisms of why CASTs have evolved these repeats remain unresolved. Moreover, all CASTs that have been identified to-date have a minimal CRISPR array with as few as two spacers. These systems are also missing the Cas1-Cas2 adaptation machinery, raising the question of how CASTs target other mobile genetic elements for horizontal gene transfer. Another open question is whether non-Tn7 transposons have adapted CRISPR-Cas systems to mobilize their genetic information. We reasoned that an expanded catalog of CASTs may shed light on many of these unresolved questions.

Here, we have systematically surveyed CASTs across metagenomic databases using a custom-built computational pipeline that identifies both Tn7- and non-Tn7 CASTs. Using this pipeline, we have identified unique architectures for Type I-B, I-F, and V CASTs. Type IF CASTs show the greatest diversity in Cas genes, including TniQCas8/5 fusions, split Cas7s, and even split Cas5 genes. Some I-F CASTs appear likely to assemble a Cascade around a short crRNA for selftargeting from a non-canonical spacer. Type I-B CASTs frequently encode two TniQ/TnsD homologs, one of which is used for self-targeting via a crRNA-independent mechanism. Remarkably, we have also found I-B systems that encode two TniQ homologs and a self-targeting crRNA, suggesting additional unexplored targeting mechanisms. In addition, we have observed new Type I-C and Type IV-family Tn7-like CASTs with unique gene architectures. Both of these sub-families lack canonical CRISPR arrays, suggesting that CASTs use distal CRISPR arrays, perhap s from active CRISPR-Cas systems, for horizontal gene transfer. We have identified multiple self-insertions and gene loss in Type V systems, indicating that target immunity - a mechanism that prevents transposons from multiple self-insertions at an attachment site-is frequently weakened. Finally, we have found a set of Cas12a-associated Rpn-family transposases that may participate in crRNA-guided horizontal gene transfer. We anticipate that these findings will shed 

available under aCC-BY-NC-ND 4.0 International license.

Rybarski, Hu and Hill et al. 28 July 2021 - preprint copy - BioRxiv

additional light on how CASTs have co-opted CRISPR-Cas systems and further expand the precision gene editing toolbox.

\section{$\underline{\text { Results }}$}

\section{A bioinformatic CRISPR-associated transposon (CAST) discovery pipeline}

We developed a bioinformatics pipeline that first searches metagenomic contigs for transposases using protein BLAST (BLASTP) and a curated transposase database with a permissive e-value threshold of $10^{-3}(11)$ (Figure 1A). All possible open reading frames (ORFs) in a 25 kilobasepair (kbp) neighborhood up-and downstream of each putative transposase are translated and searched with BLASTP using a second curated database of all Cas proteins. Contigs without Cas genes are not analyzed further. The remaining contigs contain both a transposase and at least one Cas gene. We identify CRISPR arrays in these contigs using a modified version of PILER-CR that can locate arrays with as few as two repeats (12). A final round of protein annotation searches for accessory transposase subunits (i.e., TnsC/D/E for the Tn7 family) and genetic elements that are near common attachment sites $(13,14)$. Finally, we filter the contigs by constraints (detailed in later sections) that are designed to isolate novel CASTs.

To test this pipeline, we searched for all previously discovered CASTs in the National Center for Biotechnology Information (NCBI) repository of bacterial genomes $(2,15,16)$. We downloaded 951,491 partial and complete bacterial genomes from the NCBI FTP server on May 5, 2021. Using these genomes as input, we identified regions with at least one Cas gene within a $\sim 25 \mathrm{kbp}$ neighborhood of a transposase. We filtered for contigs that include either a Type I or Type V effector and at least one member of the TniQ/TnsD family of Tn7-associated proteins. As expected, we re-identified previously published systems, along with previously unannotated Type V systems. These results confirm that the bioinformatics pipeline is sufficiently sensitive to discover these rare CRISPR-transposon systems in large genomic databases.

Next, we searched the EMBL-EBI repository of metagenomic sequencing reads for novel CASTs (17). This repository is the largest collection of sequenced DNA from diverse microbiomes, aquatic, manmade, and soil environments. We downloaded $>1$ petabyte of non-16S rRNA reads (Figure 1B, see Methods). These reads were assembled into $\sim 10$ billion high-quality contigs ( $\sim 30$ terabytes). Contigs were annotated for transposases and cas genes as described above. Approximately 54 million contigs ( 150 gigabytes) met the twin criteria of having at least one transposase and one cas gene. After de-duplication of nearly identical contigs, we searched for putative CAST systems (Figure 1B). Using Class 1 Tn7-associated CASTs as an example, we filtered for contigs that included at least one cas gene, tnsD/tniQ, and had at least one of the core $\mathrm{Tn} 7$ genes: tns $\mathrm{A}, \mathrm{tnsB}$, or tns $\mathrm{C}(\sim 1.8 \mathrm{M}$ contigs). These systems were additionally filtered by selecting only systems where two of the Tn7 genes were less than $1500 \mathrm{bp}$ apart, and where three of the core Class 1 cas genes (cas5, cas6, cas7, and cas8) were also less than 1500 bp apart (1167 systems). Of these, 1093 systems were identified as being Type IF CASTs. The remaining high-confidence systems included Type I-B, I$\mathrm{C}$, IV, and V systems (Figure 1C). To increase the confidence of our annotations, and to annotate unknown ORFs, we re-BLASTed all possible reading frames against the UniProtKB/TrEMBL database of high-quality computationally annotated protein variants (18). We assigned high confidence systems to specific sub-types by re-BLASTing the cas genes against a database of subty pe-specific effector proteins and by manually reviewing the operon architecture. After removing nearly redundant systems, we found 1476 high-confidence CRISPR-Tn7 CASTs. Notably, we detected founding members of the Type I-C and IV
CASTs in the metagenomic but not NCBI database (see below). All of these systems were missing the interference (Cas3) and adaptation (Cas 1/2) genes, in agreement with the 'guns for hire' hypothesis for how

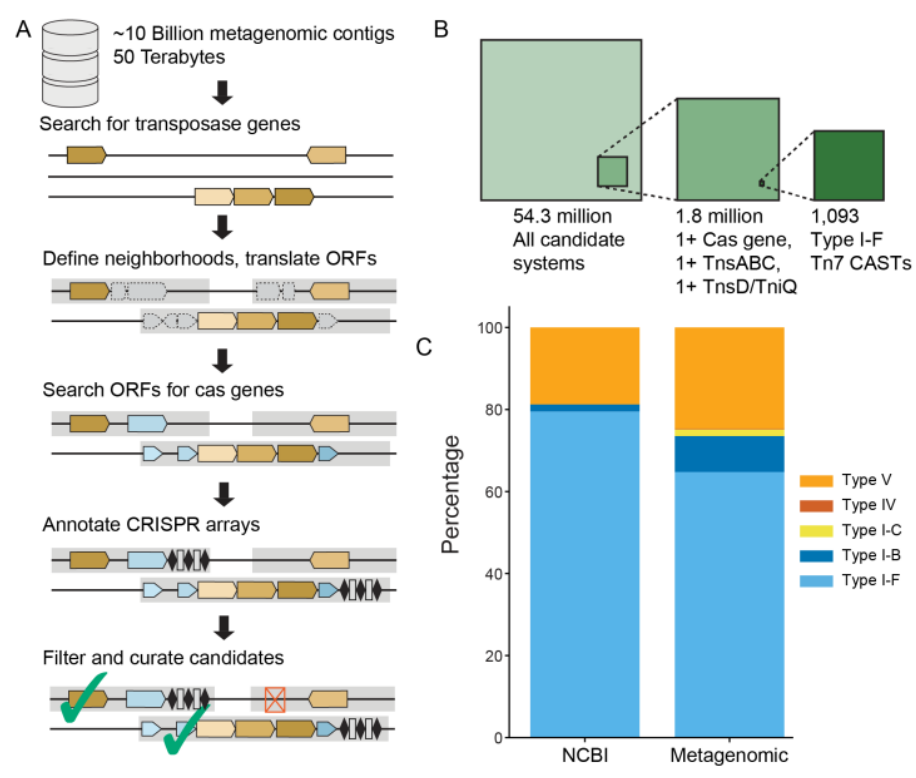

Figure 1. CRISPR-associated transposon detection and classification. (A) A bioinformatic pipeline for the discovery of CRISPR-associated transposons (CASTs). Brown: transposase genes; blue: cas genes; dotted: open reading frames; gray: gene neighborhoods. Neighborhoods satisfying initial search criteria are marked w ith a green check. Red " $x$ " denotes a neighborhood that doesn't match initial search criteria (e.g., no detected cas genes). (B) Summary of the stepw ise filtering strategy to identify high-confidence Type I-F Tn7 CASTs. (C) Distribution of Tn7associated CAST subtypes in the NCBI microbial genome and EMBL metagenomic databases.

CRISPR-Cas systems have been co-opted for diverse cellular functions (19).

\section{Diversity of Type I-F CASTs}

We identified 1093 non-redundant I-F sub-sy stems with the prototy pical gene arrangement of TnsA-TnsB-TnsC separated from TniQ-Cas8/Cas5Cas7-Cas6 by a large cargo region (Figure 2A). Tn7 cargo genes are unrelated to the transposition mechanism, and often include antibiotic resistance genes (20). Type I-F3a sy stems, defined as using the conserved genes guaC or yciA as their attachment site, comprise $\sim 61 \%$ of all I-F CASTs (Figure 2B). I-F3b sy stems, which use the rsmJ or ffs attachment site, comprise $\sim 34 \%$ of I-F CASTs (21). The remaining $5 \%$ of I-F systems form a distinct group, termed I-F3c, with a unique attachment site and self-targeting mechanism (see below).

The most common gene arrangement in our dataset for all three subtypes encodes the TnsA-C proteins in one operon, and the TniQ and Cas proteins in a second operon that is adjacent to the CRISPR repeats. A large cargo spanning $\sim 10-20 \mathrm{kbp}$ either separates these operons or is present downstream of the cas genes. The stoichiometry of the Cascade effector has been previously reported to be (Cas6) 1 : (Cas7)6: $\left(\right.$ Cas8/Cas5) 1 : crRNA $_{1}$ : TniQ 2 , based on cryo-electron microscopy of Type I-F CAST complexes $(3,4,6,22)$. In these studies, TniQ interacts with Cas7 and is structurally distant from Cas8/Cas5. However, in two of our new I-F3a systems, TniQ is expressed as an $\mathrm{N}$-terminal fusion with 
Cas8/Cas5. Four distinct I-F3a systems also have a split Cas7, and in one system, both the Cas5 and Cas 7 proteins are split into two distinct polypeptides.

All I-F3 systems that we identified appear to use a crRNAguided self-targeting mechanism that directs Cascade near the attachment site (21). The self-targeting crRNA is either in the leader-distal position of the CRISPR array or 80-85 nt away from the CRISPR array, as reported previously (21). These self-targeting crRNAs are flanked by an aty pical direct repeat that has several substitutions relative to the direct repeats within the CRISPR array. I-F3c CASTs attach upstream of a protein of unknown function that encodes seven putative transmembrane regions (see Methods). This attachment site has not been previously reported for any Tn7-family transposon. To determine how Type I-F3c systems use crRNA-guided transposition, we aligned the region around the CRISPR array with the sequence $500 \mathrm{bp}$ upstream of tnsA. This identified a short $20 \mathrm{bp}$ sequence immediately after the final canonical CRISPR repeat that matched the region approximately 64 bp upstream of the transposon end. This short, aty pical spacer is followed by an atypical repeat (Figure 2C), akin to the I-F3a and I-F3b systems.

Type I-F systems recognize a dinucleotide protospacer adjacent motif (PAM) (23). Our analysis of the self-targeting PAMs highlighted that they vary with the attachment site and CAST sub-family (Figure 2D). Next, we analyzed the sequence composition of the inverted repeats that $\operatorname{span} \operatorname{Tn} 7$. The right inverted repeat starts with a universally conserved 5'-TGT that is recognized by the essential TnsB recombinase (24). The rest of this repeat varies but is most similar between CASTs that have the same attachment site (Figure 2D). These results further confirm that I-F3c systems cluster into a distinct CAST sub-type.

\section{Type I-B CASTs encode multiple integration mechanisms}

We found four families of Type I-B CASTs that lack interference and adaptation genes. These systems either encode a single TniQ or dual TniQs of unequal length (Figure 3A). Sy stems with dual TniQs comprise
A

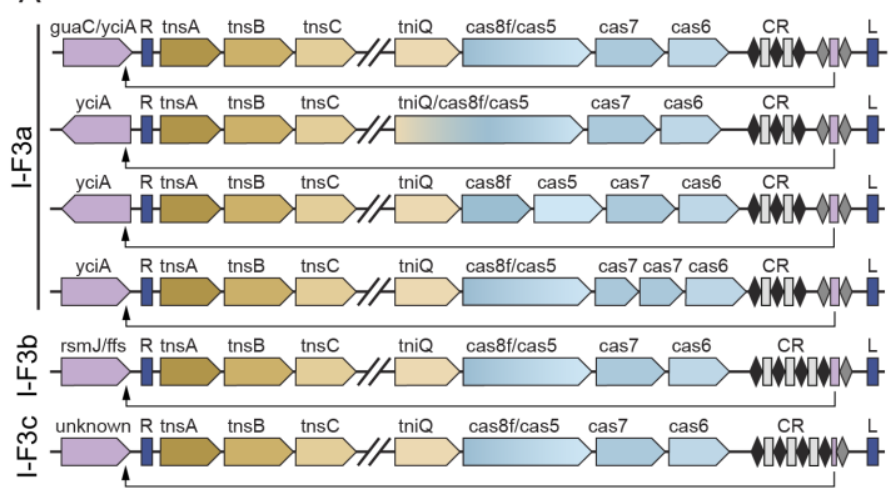

C

Repeat

Spacer

GTGTCCTGCCGAATAGGCAGCTAAGAAA TACAACTCGACCAATTTCCTTGCCGTAGTACT GTGTCCTGCCGAATAGGCAGCTAAGAAA TACCCCAATCCTTATTATTGATCTACACCGTC GTGTCCTGCCGAATAGGCAGCTAAGAAT AGGCCAGGAAGAAAGCAACC TTCCTGCTGAACAAGCAGTTAATAATAA

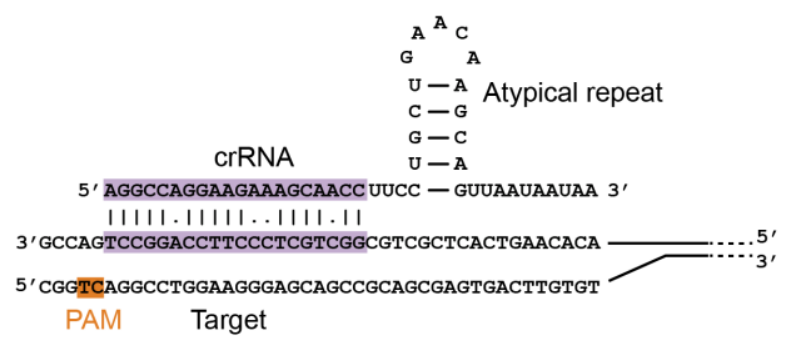

B

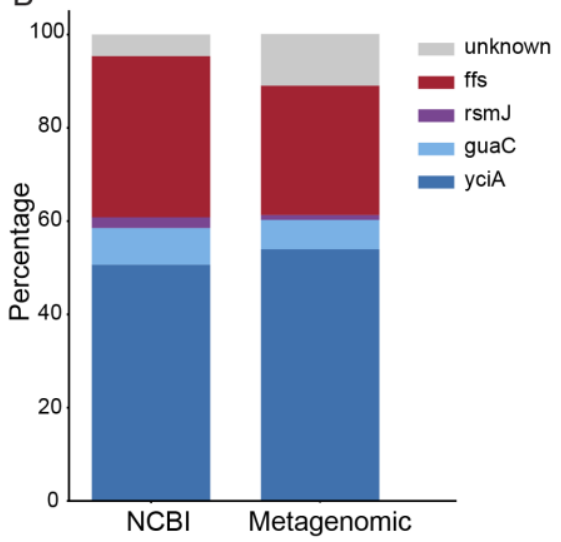

D

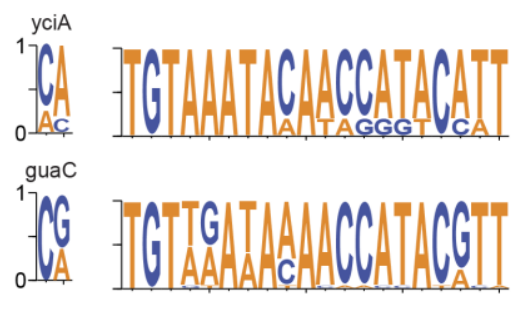

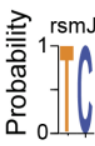

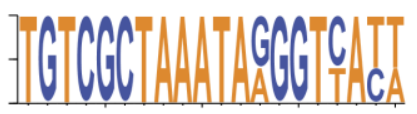

$-\sqrt{\mathrm{ffs}}$

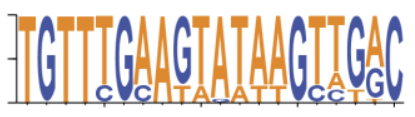

unknown

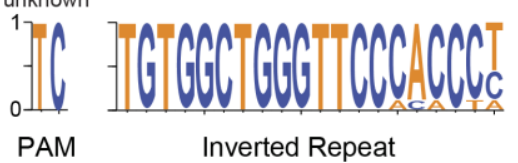

Figure 2. Summary of Type I-F CASTs. (A) Gene architectures of Type I-F3a, I-F3b, and I-F3c systems. Unique gene architectures include TniQ-Cas8 fusions, split Cas8 and Cas5, and dual Cas 7 systems. Purple: attachment site; blue: left (L) and right (R) transposon ends. Black diamonds: canonical direct repeats; gray diamonds: atypical direct repeats. Rectangles: protospacers; purple rectangle: self -targeting protospacer. Arrow indicates the target site. Slanted gapped lines indicate elided cargo regions. (B) The distribution of attachment site genes in the NCBI and the metagenomic databases. (C) (top) Sequence of a CRISPR array with a short, atypical spacer (purple) that may assemble a mini-Cascade. (bottom) Schematic of an atypical crRNA and its target DNA sequence. (D) Weblogos of the PAM and right inverted repeat adjacent to each attachment site. The TnsB binding site and the selftargeting PAMs are conserved within sub-systems. 
bioRxiv preprint doi: https://doi.org/10.1101/2021.08.16.456562; this version posted August 17, 2021. The copyright holder for this preprint (which was not certified by peer review) is the author/funder, who has granted bioRxiv a license to display the preprint in perpetuity. It is made available under aCC-BY-NC-ND 4.0 International license.

Rybarski, Hu and Hill et al. 28 July 2021 - preprint copy - BioRxiv

$79 \%$ of all identified systems. The most common Type I-B system(I-B1) encoded $\mathrm{TniQ}_{1}$ between $\mathrm{TnsC}$ and a Cas gene and $\mathrm{TniQ}_{2}$ is on the distal end of the CRISPR array (Figure 3A, top and Figure 3B). Sy stems with a single TniQ (I-B2 and I-B3) had two distinct gene architectures and self-targeting modalities. In I-B2 systems, where TniQ was sandwiched between TnsC and Cas6, we identified a self-targeting spacer that was complementary to a region downstream of glmS (Figure 3A, middle). However, we did not find any self-targeting spacers in I-B3 systems, where TniQ was between the CRISPR array and cargo genes. In addition, this TniQ was nearly double the length of the shorter TniQs found
A

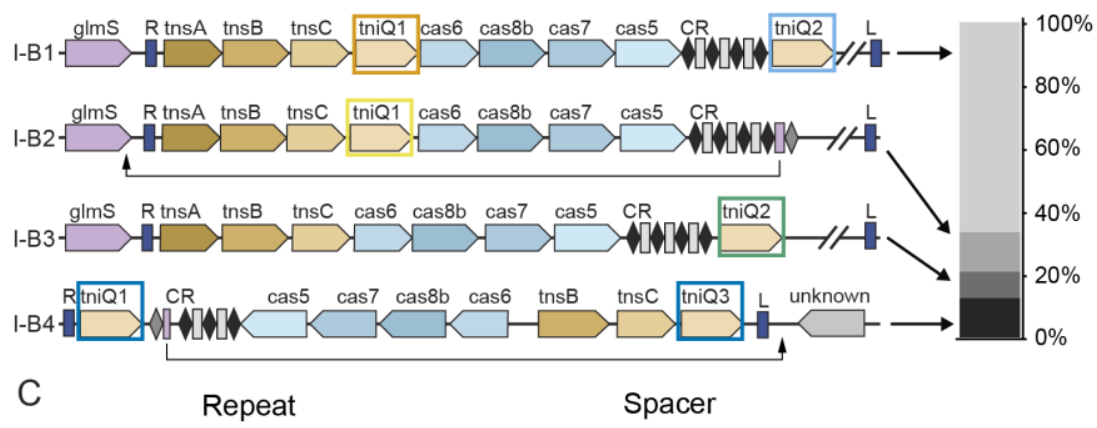

GTGCGTCATGGCTGACTGCCTATTGGCGTTGCTCA AGAAAATCGGGCTTGATTCGTTGCTGAACCCGCCTCAGTTGCTGAAACCGCGTC

GTACATCATGGTTTACTGCCTATTGGCGTTGCTCA ATACTGAACAGTTCCTTGGCTATCAATAGCAAAATAAAGCATA

GTACATCATGGTTTACTGCCTATTGGCGTTGCTCA GGGATTCGTTTACATTTTACATTTTGCCCGTTCCATT

GTGCATCATGGCTAACTGCCTAATGGCGTTATCTA
B

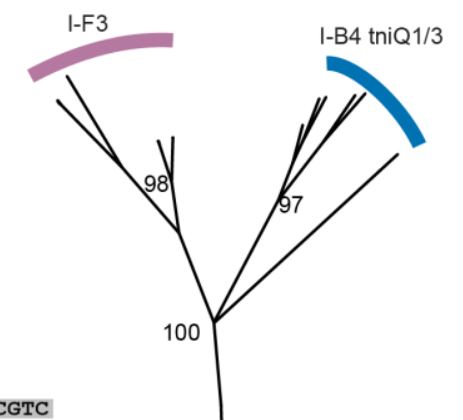

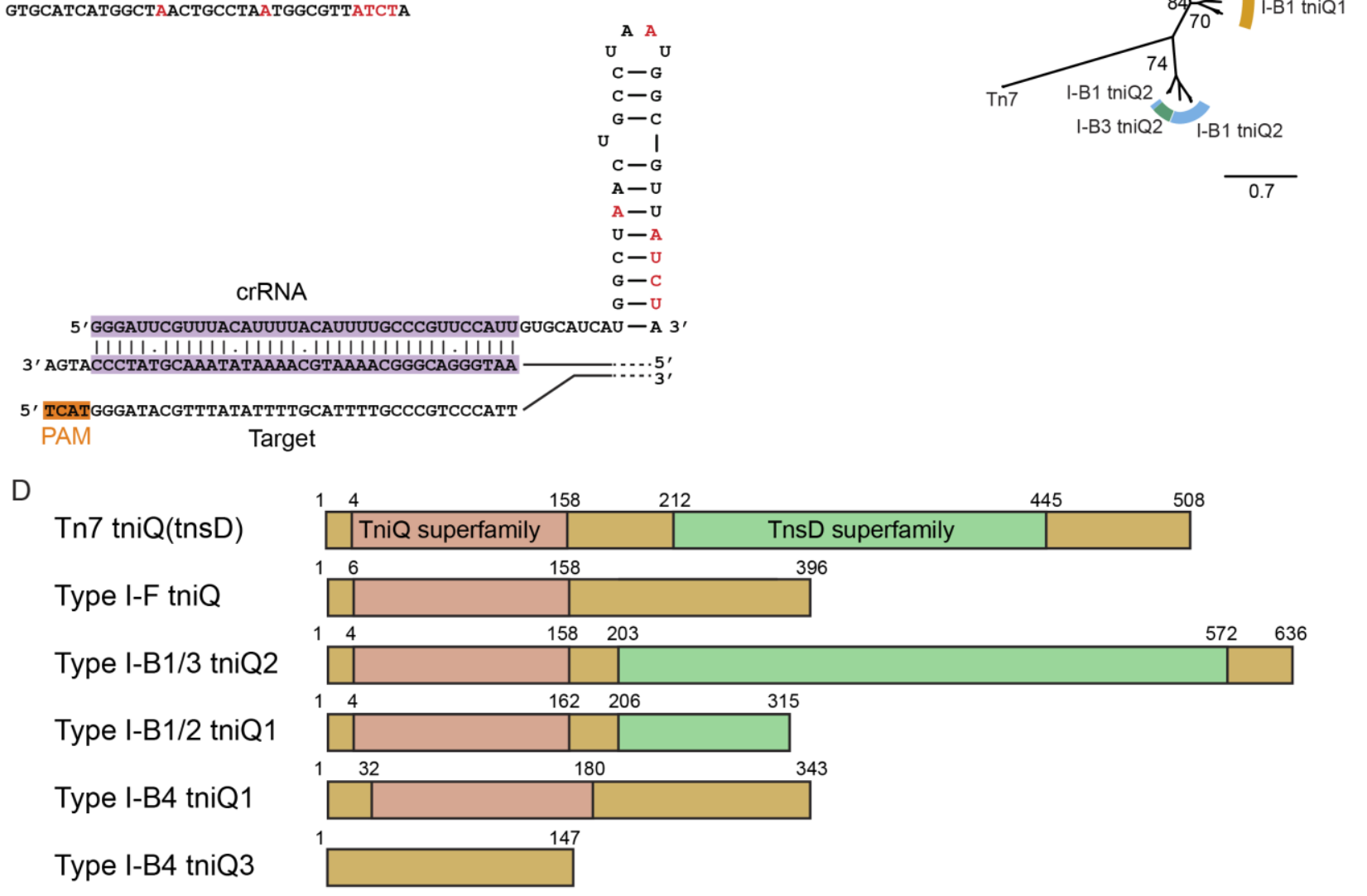

Figure 3. Analysis of Type I-B CASTs. (A) (left) Gene architectures of Type I-B systems. Systems can dispense with either the first or the second tniQ, suggesting alternative targeting lifestyles. Type I-B4 systems have a unique architecture that most resembles Type V CASTs. Colored rectangles correspond to phylogenetic groups in panel B. (right) The distribution of Type I-B sub-systems in the metagenomic database. (B) Phylogenetic tree w ith tniQ variants from Type I-B and I-F CASTs, as w ell as from the canonical Tn7 transposon. Type I-B tniQ1 is most similar to tniQ from Type I-F CASTs, w hereas tniQ2 is closely related to canonical Tn7 tnsD. Values at branch points are bootstrap support percentages. (C) (top) Sequence of a Type I-B4 CRISPR array w ith a short, atypical spacer. (bottom) Schematic of an atypical crRNA basepaired w ith a target DNA sequence. Red bases are those that differ from the canonical repeat sequence. (D) Domain maps of TniQ proteins. Regions homologous to the TniQ superfamily and the TnsD superfamily are indicated in pink and light green, respectively. The Type I-B4 systemencodes the shortest TniQ variant. 
between TnsC and Cas6 and bore a strong resemblance to the TnsD encoded in canonical Tn7 systems. Notably, we also found single-TniQ systems that encode a TnsD-like TniQ but also lack self-targeting spacers.

We identified an atypical Type I-B CAST (I-B4) that had a unique gene architecture and self-targeting mechanism (bottom row, Figure 3A). This system encodes TnsB and TnsC but lacks the TnsA gene, akin to Type V systems $(8,9)$. Two TniQ homologs of unequal length are immediately adjacent to the inverted repeats but distal from the Cas operon. TniQ ${ }_{1}$ is sandwiched between the right transposon end and a short CRISPR array; $\mathrm{TniQ}_{3}$ is only $\sim 450 \mathrm{bp}$ long and is located between $\mathrm{TnsC}$ and the left transposon end. This short $\mathrm{TniQ}_{3}$ can be aligned against the N-terminus of traditional CAST-associated TniQs (Figure 3D). Notably, this is the only dual-TniQ CAST that encodes a self-targeting spacer with near-perfect complementarity to a region of DNA just outside the left transposon end. The attachment site is adjacent to a gene of unknown function near the left transposon end (Figure 3A), akin to the attachment sites in Type V CASTs. The self-targeting spacer is flanked by an atypical direct repeat and is also 6 to 23 bp shorter than the other spacers in the CRISPR array (Figure 3C). Based on these findings, and our observation of Type I-F CASTs with short self-targeting spacers, we propose that Type I-B systems can also assemble self-targeting miniCascades.

To better understand the roles of multiple TniQs in Type I-B CASTs, we constructed a phy logenetic tree of Type I-B, I-F3a, and I-F3b TniQs, along with TnsD from the canonical Tn7 transposon (Figure 3B). Compared with the cluster of $\mathrm{TniQ}_{2}$, the short $\mathrm{TniQ}_{1}$ from Type I-B1 and B2 systems are closer to TniQ from other CASTs, while the Type I-B1 $\mathrm{TniQ}_{2}$ clusters with canonical Tn7 TnsD. The close similarity between $\mathrm{TniQ}_{2}$ and $\mathrm{Tn} 7 \mathrm{tnsD}$, along with the apparent lack of self-targeting in
A

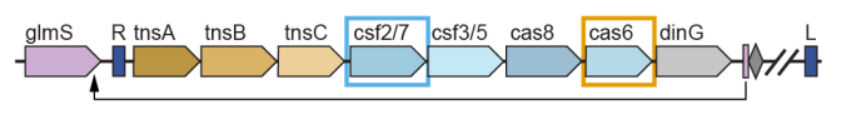

$$
\begin{gathered}
\text { U U } \\
\mathrm{A}-\mathrm{U} \\
\mathrm{U}-\mathrm{G} \\
\mathrm{U}-\mathrm{A} \\
\mathrm{A}-\mathrm{U} \\
\mathrm{C}-\mathrm{G} \\
\mathrm{I} \mathrm{A} \\
\mathrm{A}-\mathrm{U} \\
\mathrm{U}-\mathrm{A} \\
\mathrm{U}-\mathrm{A}
\end{gathered}
$$

crRNA

UAAUCAGCAACCACGUUAUCUUUACUAUUUU - GCC || . I | | | | | I I I | | | | 1
GTCGATGAGTCGATGGTGCA CAGCTACTCAGCTACCACGT PAM

$$
\text { Target }
$$

C

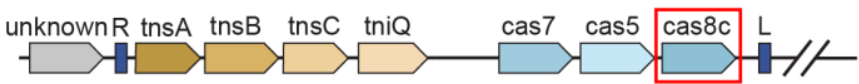

\section{Type I-C cas8}

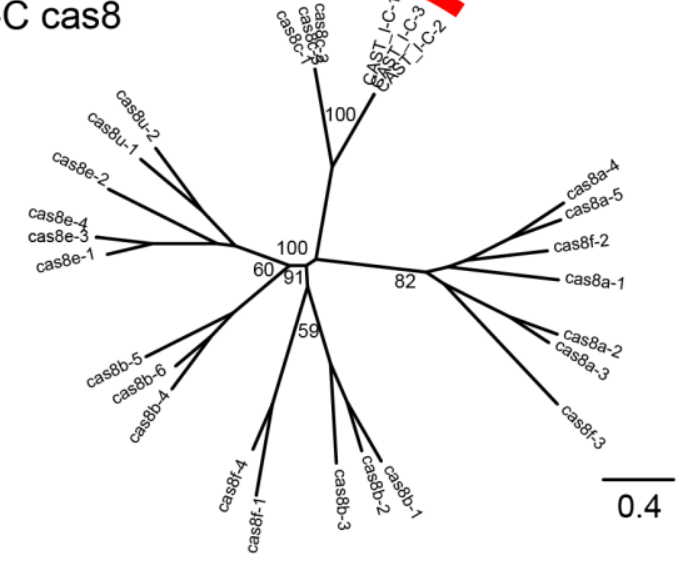

B
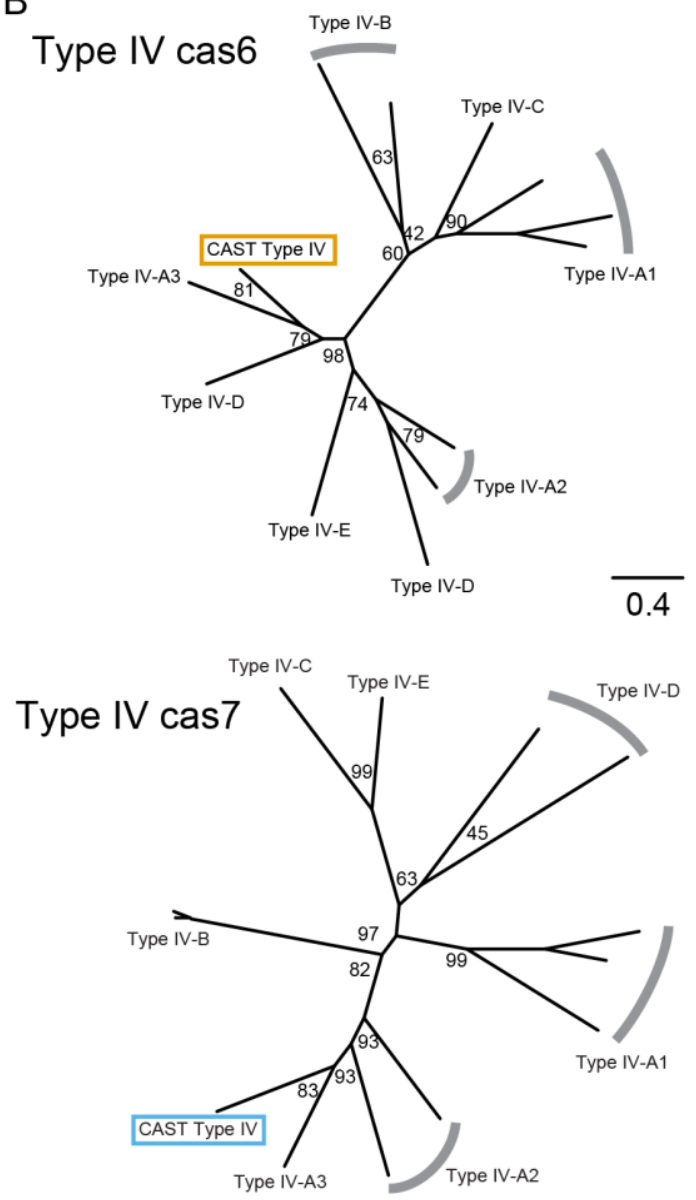

0.4

Figure 4. New Tn7 CASTs from metagenomic databases. (A) (top) Gene architecture of a Type IV CAST. This system lacks a CRISPR array but encodes a self-targeting spacer. Genes highlighted by colored rectangles correspond to genes in panel B. (bottom) Schematic of a short, self-targeting spacer basepaired w ith its target DNA sequence. (B) Phylogenetic trees of cas 6 and cas 7 indicate that the Type IV CAST most closely resembles Type IV-A3 CRISPR-Cas systems. Values at branch points are bootstrap support percentages. (C) (top) Gene architecture of Type I-C systems. We did not detect any CRISPR arrays or atypical self-targeting spacers. (bottom) A phylogenetic tree of cas 8 conf irms that this system is closely related to Type I-C Cascades. Values at branch points are bootstrap support percentages. 
Rybarski, Hu and Hill et al. 28 July 2021 - preprint copy - BioRxiv

most dual TniQ systems, suggests that $\mathrm{TniQ}_{2}$ serves the same role as TnsD, namely that it is a sequence-specific DNA-binding protein that directs transposition downstream of glmS. TniQ 1 , in turn, forms a complex with Cascade to guide TnsABC to a crRNA-directed target. While this work was in preparation, an experimental study demonstrated that I-B CASTs with two tniQ genes use two separate pathways for target selection (7).

\section{Novel Type I-Cand IV CASTs from metagenomic sources}

Our analysis of the metagenomic contigs revealed a Type IV CAST (Figure 4A). Type IV systems are primarily encoded by plasmid-like elements to mediate inter-plasmid conflicts $(25,26)$. Phylogenetic trees of Cas 6 and Cas 7 independently placed this CAST within the Type IVA3 sub-family (Figure 4B). These sy stems frequently shed their CRISPR repeats, instead of using distal CRISPR arrays (25). Although we did not find any CRISPR repeats in this system using CRASS or PILER-CR, we detected a spacer-like DNA segment with strong complementarity to the C-terminus of glmS, the likely attachment site. This putative selftargeting spacer is adjacent to a hairpin that resembles the direct repeats in other Type IV CRISPR-Cas systems (Figure 4A, bottom). We conclude that this minimal spacer-repeat motif directs self-targeting by the Type IV system. Horizontal transfer may still occur via a distal CRISPR array, akin to the interference mechanism in other Type IV CRISPR-Cas systems (25).

We found nine non-redundant Type I-C CASTs with Cas5, Cas7, and Cas8 downstream of TnsABC and TniQ (Figure 4C). TniQ is immediately adjacent to TnsABC rather than the Cas proteins. A phy logenetic analy sis of Cas 8 showed close similarity to Cas $8 \mathrm{c}$ (Figure 4C, right). We did not detect a CRISPR array via CRASS or PILER-CR. We also did not detect any tRNA or common Tn7-associated attachment sites near either the left or right transposon ends, precluding a detailed analysis of the self-targeting mechanism. We cannot rule out that these systems use a minimal leader-spacer array that was below the threshold of our detection software. Alternatively, these systems may use a distal CRISPR array in trans. Further bioinformatic and experimental analy ses will be required to delineate the mechanisms of self-targeting and horizontal transfer in these sy stems.

\section{Architectural diversity and self-targeting in Type V CASTs}

Type VCASTs were likely formed when a Tn7-like transposon co-opted a Cas 12 gene for RNA-guided DNA targeting (10, 15). Most Type V CASTs contain TnsB, TnsC, and TniQ at one end of the transposon with Cas 12k, a small CRISPR array, and an aty pical repeat-spacer on the other end. Cargo genes spanning 2 to $23 \mathrm{~kb}$ of additional DNA sequences are sandwiched between TniQ and Cas 12k (Figure 5A). In contrast to the Class 1 systems described above, all metagenomic Type V systems lacked TnsA, consistent with the proposal that Cas $12 \mathrm{k}$ was captured by a Tn5053-family transposon, which contains TnsB, TnsC, and TniQ homologs but also lacks TnsA (27). The tracrRNA is upstream of the canonical CRISPR array with homology to the atypical repeat. The crRNA usually has good homology to the target DNA, with $98 \%$ of systems containing one or zero mismatches in the first ten basepairs (Figure 5B). As previously observed (8), atypical spacers generally targeted tRNA genes immediately adjacent to the transposon. We also found one system that attaches $104 \mathrm{bp}$ downstream of ArsS, an arsenosugar biosynthesis radical SAM protein. Analysis of the DNA upstream of the self-targeting spacer revealed 5'-TGGTA as the most common PAM, with some variability in the $-5,-4$, and -1 positions. (Figure 5C). Experimental evidence for two CASTs showed a preference for a smaller 5'-GTN PAM (9). Whether the broader set of Cas 12k proteins have more stringent PAM requirements will require experimental validation. Overall, this architecture and preference for tRNA attachment sites corroborate previous bioinformatic and experimental observations $(7-9,15)$.

We also found Type V CASTs with unusual TnsC and TnsB arrangements. Notably, all Type V TnsC proteins lack the canonical TnsA- and TnsB-interacting domains and have partial truncations of the TniQ-interacting domain (28-30). The shortest CAST - only $6.6 \mathrm{kbp}$ in total, including the cargo-encodes a 98 amino acid TnsC fragment whose sequence overlaps with TnsB by 115 bp (Figure 5A, 5D). In addition to losing the TniQ, TnsB, and TnsA interacting domains, this TnsC has also lost its ATPase domain $(28,30)$. We hy pothesize that the minimal TnsC encodes uncharacterized TnsB- and TniQ-interaction motifs. Because of its compact organization, this CAST is also a prime candidate for gene editing applications.

Multiple CASTs split TnsB into separate ORFs that encode just the $\mathrm{N}$ - or C-terminus. Alternatively, a full-length TnsB is encoded next to a TnsB fragment containing most of the cataly tic domain (Figure 5E). Strikingly, two unrelated systems encode the same $\mathrm{N}$-terminal region of TnsB. We speculate that these split TnsBs form a heterodimeric $\mathrm{TnsB}_{1}: \mathrm{TnsB}_{2}$ transposition complex. These heterodimeric complexes retain the catalytic core while also maintaining the requisite TnsC interaction motifs via the longer TnsB subunit.

We also observed multiple contigs with two CASTs inserted at the same attachment site (bottom rows, Figure 5A). Tn7 transposons can prevent re-insertion at the attachment site by TnsB-mediated dissociation of TnsC from target DNA $(31,32)$. However, more distant Tn7-family transposases may still insert at a single attachment site, resulting in several transposons that are situated adjacent to each other (30). Consistent with this idea, the dual-insertion Type V CASTs have distinct cargos, unique gene architectures, and divergent Cas $12 \mathrm{k}$ sequences. In one scenario, the tRNA-distal CAST encoded an Nterminal TnsB truncation and lost TniQ (7th row, Figure 5A). The tRNAproximal CAST from the same organism encoded the C-terminal TnsB fragment and had a complete Type V-family TniQ. A second dual CAST system had lost both TnsC and TniQ from the tRNA-distal transposon (last row, Figure 5A). Both sy stems encode a self-targeting spacer and a full Cas $12 \mathrm{k}$ gene, suggesting that they are both still active.

\section{Phylogenetic analysis of Tn7-CASTs}

To clarify the evolutionary relationship between Tn7-CASTs, we built phylogenetic trees of the TnsB (Supplemental Figure 1A) and TnsC (Supplemental Figure 1B) proteins from all known CAST subtypes as well as Tn7-family transposons (see Methods). The phylogenetic relationships between sub-systems were nearly identical for both TnsB and $\mathrm{TnsC}$, suggesting that these proteins are co-evolving as a system. We omitted TnsA from this analysis because Type I-B4 and all Type VTn7CASTs lack this gene. We confirmed that all metagenomic Type VCASTs are phylogenetically closer to the Tn5053 transposon than Tn7. In contrast, Type I-B1-3, I-C, and IV CASTs are phylogenetically close to $\mathrm{Tn} 7$. Such limited evolutionary drift may suggest a relatively recent co-opting of this CRISPR-Cas system. Type I-B4 CASTs are a notable exception because these systems also lack TnsA and cluster closer to Tn5053 than to the reference Tn7. Type I-F CASTs are highly divergent from both $\operatorname{Tn} 7$ and Tn5053, with a large phy logenetic separation between the I-F3a and I-F3b sub-types. The diversity of Tn7-family transposases and CRISPR sub-systems that have co-evolved suggests that we will continue to find new CAST families as metagenomic databases expand and detection sensitivity increases. 
bioRxiv preprint doi: https://doi.org/10.1101/2021.08.16.456562; this version posted August 17, 2021. The copyright holder for this preprint (which was not certified by peer review) is the author/funder, who has granted bioRxiv a license to display the preprint in perpetuity. It is made available under aCC-BY-NC-ND 4.0 International license.

Rybarski, Hu, and Hill et al., 28 July 2021 - preprint copy - BioRxiv

A

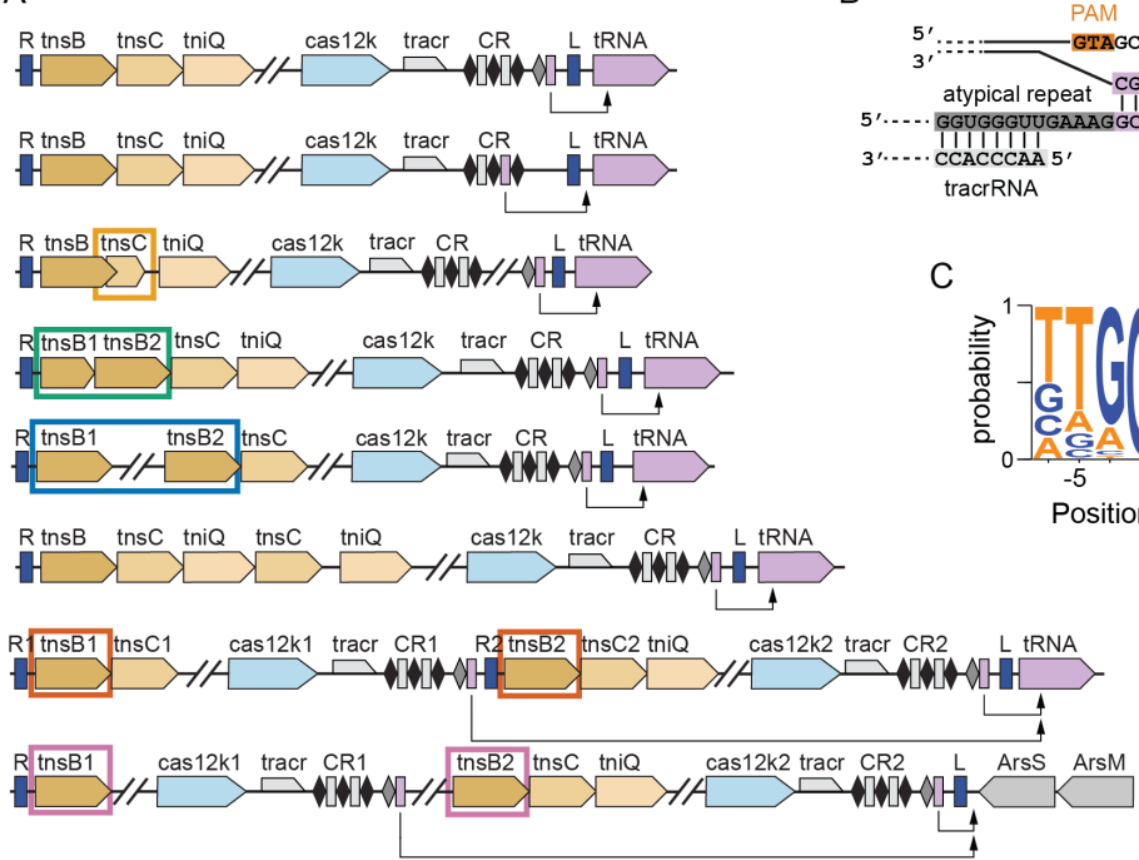

D

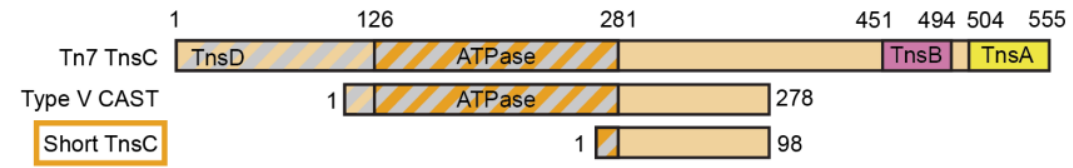

$\mathrm{E}$

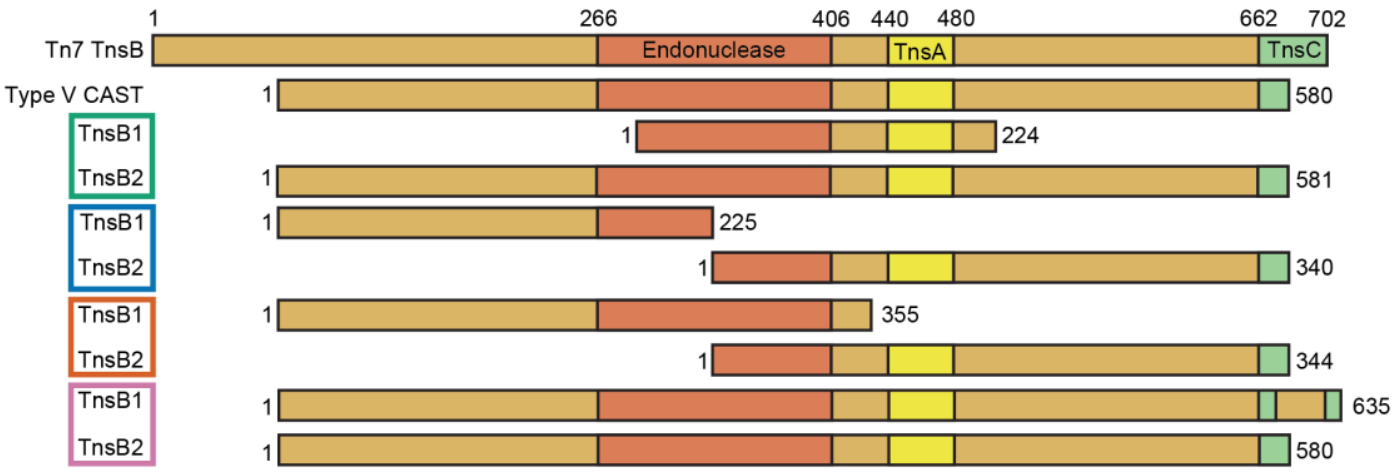

Figure 5. Analys is of Type V CASTs. (A) Gene architectures of Type V CASTs, including dual-insertion systems (bottomtwo rows). Colored rectangles around genes correspond to alignments in panels $D$ and $E$. (B) Schematic of interactions betw een the target site DNA, a self-targeting crRNA, and a tracrRNA. (C) Weblogo of PAM sequences found adjacent to spacer targets. (D) Aligned domain maps of truncated Tns $\mathrm{C}$ variants. Gray diagonal stripes indicate TnsD-interacting region. Truncated TnsCs lack the TnsA-and TnsBinteracting domains but generally retain the ATPase domain and most of the TnsD-interacting domain. The shortest TnsC has also lost its ATPase domain. (E) Aligned domain maps of truncated TnsB variants. Type V CAST TnsB is shorter than Tn7 TnsB but contains the functionally annotated domains. In some dual TnsB systems, the first TnsB encodes the $\mathrm{N}$-terminal region and the second encodes the C-terminal portion.

\section{Non-Tn7 CASTs that co-opt Cas12a and Type I-E Cascade}

While transposons other than Tn7 may have co-opted CRISPR-Cas systems for attachment site recognition, this has never been reported. To explore this possibility, we identified contigs that encode: (1) at least one non-Tn7 transposase gene (see Methods), (2) a CRISPR array containing at least two spacers, and (3) Class 1 or Class 2 DNA-binding effectors (i.e., Cas9, Cas 12 , Cas 13 , or any three of Cas5/6/7/8). We excluded contigs that encode interference (i.e., Cas3 or Cas10) or acquisition 
bioRxiv preprint doi: https://doi.org/10.1101/2021.08.16.456562; this version posted August 17, 2021. The copyright holder for this preprint (which was not certified by peer review) is the author/funder, who has granted bioRxiv a license to display the preprint in perpetuity. It is made available under aCC-BY-NC-ND 4.0 International license.

Rybarski, Hu and Hill et al. 28 July 2021 - preprint copy - BioRxiv

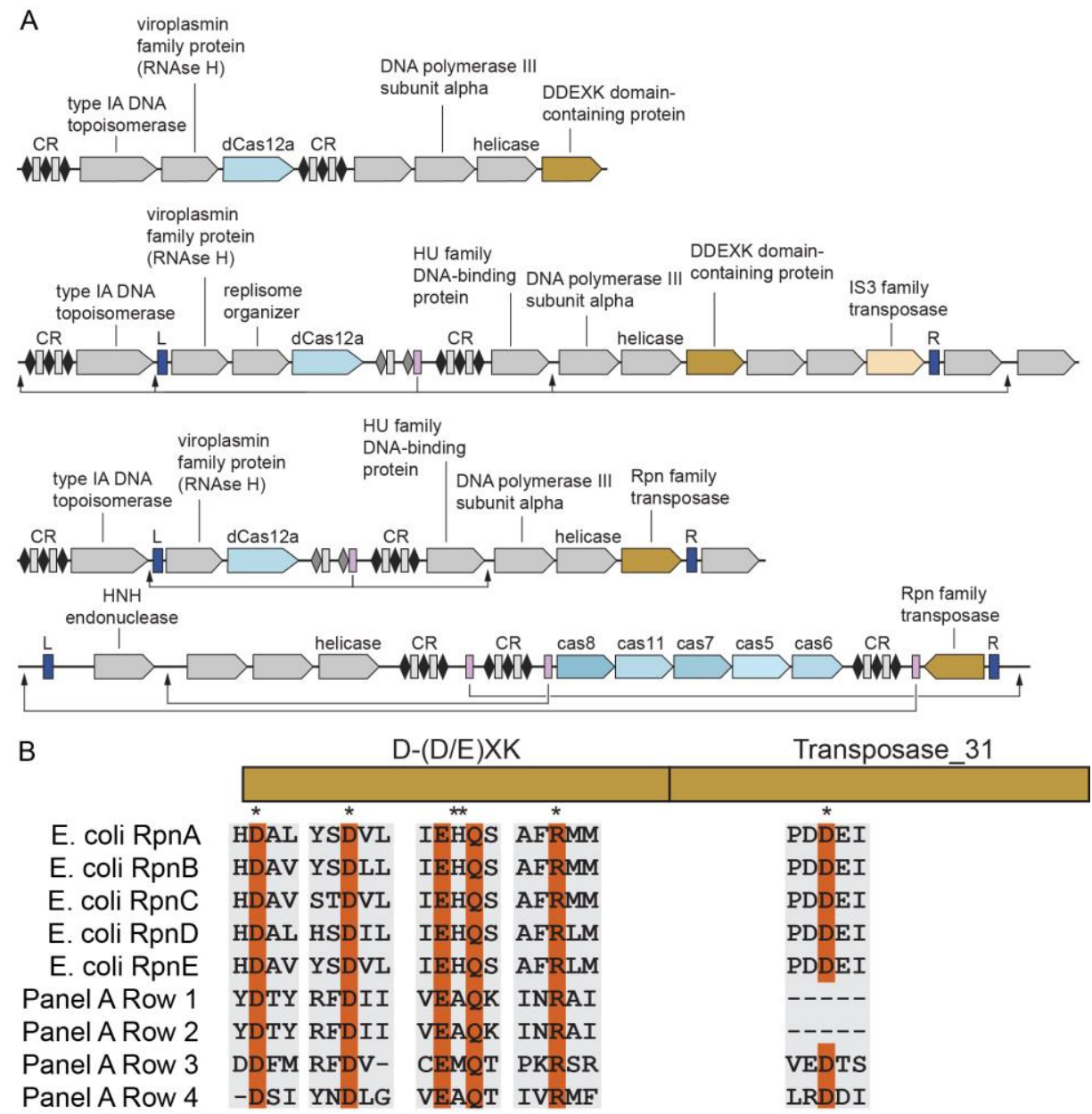

C

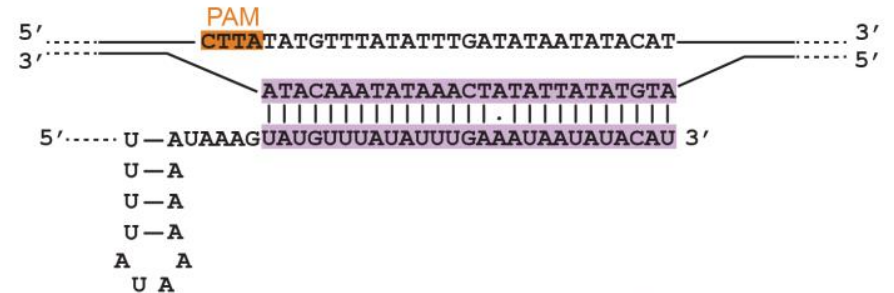

D

\begin{tabular}{|rc|c|c|c|}
\hline & \multicolumn{5}{c}{ WED RuvC } \\
AsCas12a & MAHRL & LNKKL & IIKDR & GIDRG \\
FnCas12a & ITHPA & ANKNK & LIKDK & SIDRG \\
LbCas12a & VVHPA & ANKNP & VYKDK & GIDRG \\
Panel A Row 1 & DVHPV & VNKNT & HIKDK & AVIRG \\
Panel A Row 2 & DKHPA & VNKKT & HIKDK & SVIRG \\
Panel A Row 3 & DKHPA & INKKT & HIKDK & SVIRG
\end{tabular}

Figure 6. A family of putative non-Tn7 CASTs. (A) The def ining features of this family of systems are an Rpn-family (PDDEXK domain-containing) nuclease/transposase near a nuclease-dead Cas 12 a or a Type I-E Cascade complex. The operon is enriched for nucleic-acid processing proteins. We also observed self-targeting spacers (magenta, black arrows) and short inverted repeats (blue) in some systems. (B) Multiple sequence alignment of Rpn proteins $w$ ith the putative transposases from these systems. Residues critical for DNA cleavage in the PDDEXK domain are highlighted in red. The D165A mutant in RpnA more than doubles recombination in vivo; this aspartic acid is highlighted in red below the transposase_31 domain. (C) Schematic of an atypical self-targeting spacer and its DNA target. The PAM is highlighted. (D) Multiple sequence alignment of nuclease-active Cas12a and putative CAST Cas12as. Putative CAST Cas12as retain the conserved residues in the WED domain that are essential for crRNA processing, but lack an aspartic residue in the RuvC domain that is essential for DNA cleavage. 
Rybarski, Hu, and Hill et al., 28 July 2021 - preprint copy - BioRxiv

filtered by size to exclude truncated genes (see Methods). We prioritized Type II, V, and VI systems where the cataly tic nuclease domain residues are mutated or deleted, as these enzymes cannot participate in adaptive immunity. We detected nuclease-inactivating mutations or deletions in $25 \%$ of Cas9 genes (in one or both nuclease domains), $8 \%$ of Cas 12 genes, and none of the Cas 13 genes.

We found 40 non-redundant examples of a nuclease-inactive Cas 12a or a Type I-E Cascade near a putative recombination-promoting nuclease/transposase (Rpn)-like protein (Figure 6A). Rpn family proteins were originally investigated because of their close homology to the catalytic domain of transposase_31 (33). These proteins contain a PDDEXK nuclease domain, first discovered in restriction endonucleases, but also observed in T7 TnsA and other diverse DNA-processing enzymes (34-36) (Figure 6B). E. coli RpnA promotes RecAindependent gene transfer in cells and is a $\mathrm{Ca}^{2+}$-stimulated DNA nuclease in vitro (33). The mechanism of how RpnA promotes horizontal gene transfer is unknown.

The genetic context around Cas $12 \mathrm{a}$ in our systems is highly enriched with nucleic acid-interacting proteins, including a topoisomerase, an RNAse, a DNA polymerase subunit, and one or two helicases. Likewise, the Cascade sy stem encodes a helicase and an $\mathrm{HNH}$ endonuclease. Three Cas 12a sy stems encode an HU family DNA-binding protein, and one of those also contains a protein with homology to a phage replisome organizer (37). We detected three systems with putative atypical self-targeting spacers adjacent to a canonical CRISPR array (Figure 6A). In the Cas12a systems, the self-targeting spacer is complementary to two or four nearby targets, all of which are positioned at intergenic sequences. The closest targets of these self-targeting sequences are adjacent to a 5'-CTTA PAM, which is recognized by conventional Cas 12a nucleases (Figure 6C) $(38,39)$. There are 9 bp inverted repeats (with one mismatch) that flank Cas $12 \mathrm{a}$, the Rpn-family protein, and several other genes.

The Cas 12 a genes in these systems cover $90 \%$ of the wellcharacterized AsCas 12a sequence ( $24 \%$ amino acid identity), including the critical crRNA-processing, DNA binding, and nuclease domains (40). Cas 12a can process its own pre-crRNA via a dedicated RNAse domain (41). Three residues in this domain have been identified as critical for pre-crRNA processing; all are conserved in Rpn-associated Cas12a variants (Figure 6D) (41). Cas12a degrades double-stranded DNA by first cleaving the non-target strand, followed by the target strand in its single RuvC nuclease active site (42-45). Phosphate bond scission is catalyzed by two magnesium ions, one of which is coordinated by a critical aspartic residue (position 908 in Acidaminococcus (As) Cas 12a). This residue is mutated to isoleucine in all Rpn-associated systems (Figure 6D). Similarly, the Type I-E system encodes all the Cascade subunits but does not have Cas3. We conclude that these systems bear striking resemblance to Tn7-associated CASTs and may also mobilize genomic information for crRNA-guided horizontal transfer.

\section{Discussion}

CASTs are rare in fully-sequenced prokary otic genomes and are likely to be missed by traditional CRISPR detection pipelines due to their unusual operon structures and short CRISPR array s. To address this gap, we have developed a set of Python libraries that allow users to efficiently use BLAST to search for co-occurring genes and to perform subsequent searches for arbitrary gene architectures. We have examined approximately 30 terabytes of metagenomic contigs to identify $\sim 1476$ high-confidence CASTs, including novel Type IV and Type I-C systems, as well as a Type I-B4 CRISPR system that co-evolved with a Tn5053- like element, a member of the Tn7 family of transposons that lacks TnsA. We have also discovered systems that include a putative nucleaseinactive Cas12a and a non-Tn7 transposase-like recombinase. The diversity of CRISPR sub-types that have been co-opted by transposons will likely increase as metagenomic databases and sensitive detection pipelines continue to improve $(46,47)$. More broadly, the abundance of nuclease-inactive CRISPR-Cas operons may suggest that the scope of cellular processes that have co-opted CRISPR-Cas sy stems is wider than is currently known.

In the NCBI and metagenomic databases, the most abundant Tn7-associated CASTs are those that co-opt Class 1 CRISPR systems. Of these, Ty pe I-F sub-systems are the most structurally diverse. Notably, we found that some I-F CASTs encode TniQ-Cas8/Cas5 fusions, duplicate Cas $5 \mathrm{~s}$, and duplicate Cas $7 \mathrm{~s}$. We speculate that gene duplication of the Cas 5 and/or Cas 7 allowed one of the paralogs to form a proteinprotein interface with TniQ. The second paralog may have been subsequently lost. The remaining paralog resulted in the streamlined I-F CASTs that are most frequently found in bacterial genomes. The short aty pical pre-crRNA in some I-F CASTs also suggests that these sy stems assemble a shortened Cascade for self-targeting. The size of Type I-E and I-F Cascades can be tuned by the length of the crRNA (48-51). Intriguingly, short I-F Cascades cannot recruit Cas 3 but are still able to bind the target DNA, making them an ideal system for directed Tn7 transposition (51). Short Cascades, along with the aty pical direct repeats, may differentiate self-targeting CASTs from those undergoing horizontal gene transfer in the I-F3c system.

Type I-B CASTs encode one or two TniQ/TnsD homologs. A recent report has uncovered that self-targeting in some systems proceeds via TnsD, whereas horizontal transfer is crRNA-guided (7). We also identified atypical systems that encode two short TniQ homologs along with a self-targeting spacer. Both homologs in the atypical dual-TniQ systems are related to the I-F CAST TniQ. Based on this observation, along with the crRNA-directed self-targeting, and the alignment of $\mathrm{TniQ}_{3}$ to the $\mathrm{N}$-terminus of $\mathrm{TniQ}_{1}$, we propose that this CAST assembles a hetero-dimeric Cascade consisting of a single repeat of each subunit. Alternatively, this system may assemble $\mathrm{TniQ}_{1^{-}}$and $\mathrm{TniQ}_{3}$-only Cascades for self-targeting and horizontal transfer. Additional studies will be required to decipher the role of $\mathrm{TniQ}_{3}$ in the aty pical I-B sy stems with a self-targeting spacer.

How do CASTs target mobile genetic elements with minimal CRISPR arrays? We did not find any systems that retained the Cas 1/Cas2 acquisition machinery, suggesting that strong evolutionary pressure is preventing the CAST-associated CRISPR arrays from expanding. CASTs encode CRISPR arrays that are significantly shorter than the corresponding canonical CRISPR-Cas systems and these arrays may also be transcriptionally silenced via $x$ re elements that are frequently found adjacent to these arrays in CASTs (21). Moreover, we could not identify any CRISPR arrays in Type IV and I-C CASTs. We propose that CASTs use CRISPR arrays that occur elsewhere in the genome-perhaps in functional CRISPR-Cas sy stems - for horizontal gene transfer. Evidence for such in trans CRISPR array usage has already been documented for a large set of canonical CRISPR-Cas systems $(52,53)$. CRISPR arrays that are associated with active interference machinery serve as an everupdating record of the most likely mobile genetic elements that the CAST can use for horizontal gene transfer (54). A second possibility is that Cas 1/2/4 from an active CRISPR-Cas system can act in trans to add spacers to the CAST CRISPR array. This may be an important secondary mechanism when horizontal transfer places the CAST into a host that lacks a compatible CRISPR array.

Our search revealed Cas12a proteins associated with Rpnfamily transposases, two of which appear to have atypical spacers that target two sites up - and downstream of Cas 12a. This curious arrangement 
Rybarski, Hu and Hill et al. 28 July 2021 - preprint copy - BioRxiv

could be the result of a duplication of the target site that is originally present in only a single copy. We note that the HU family DNA-binding protein is only present in systems with putative self-targeting spacers and that a homolog of this protein is essential in bacteriophage $\mathrm{Mu}$ for transpososome assembly (55). Two other proteins of viral origin - a replisome organizer and a ribonuclease - are also found near Cas 12a in a self-targeting system, hinting at an intriguing evolutionary path for the creation of this putative CAST. In sum, the CAST identification pipeline and diversity of new systems described herein add to our understanding of CRISPR transposons, expand the gene-editing toolkit, and hints at the possibility that nuclease-inactive Cas genes may play additional roles in cellular DNA metabolism.

\section{Materials and Methods}

\section{Database acquisition and contig assembly}

NCBI genomes were downloaded using NCBI Genome Downloading Scripts (https://github.com/kblin/ncbi-genome-download) on May 5, 2021, with the commands:

ncbi-genome-download --formats fasta bacteria

ncbi-genome-download --formats fasta archaea

Raw FASTQ files were downloaded from the EMBL-EBI repository (17) of metagenomic sequencing at ftp://ftp.ebi.ac.uk/vol1/ between January and February 2020. For each sample, the quality of the raw data was assessed with FastQC (56) using the command:

fastqc tara_reads_*.fastq.gz

Low quality reads were trimmed with Sickle (57) using the command:

sickle pe

-f name_reads_R1.fastq.gz

-r name_reads_R2.fastq.gz

-t sanger

-o name_trimmed_R1.fastq

-p name_trimmed_R2.fastq

-s /dev/null

We then used Megahit (58) to assemble the trimmed data with:

megahit

-1 name_trimmed_R1.fastq

-2 name_trimmed_R2.fastq

-o name_assembly

\section{Analysis pipeline}

We developed a Python library, Opfi (short for Operon Finder), to search genomic or metagenomic sequence data for putative CRISPR transposons. This library consists of two modules, Gene Finder and Operon Analyzer. The Gene Finder module enables the user to use BLAST to identify genomic neighborhoods that contain specific sets of genes, such as Cas9 or TnsA. It can also identify CRISPR repeats. The Operon Analyzer module further filters the output from Gene Finder by imposing additional user-defined constraints on the initial hits. For example, Operon Analyzer can be used to find all genomic regions that contain a transposase and at least two Cas genes but no Cas3.

We used Gene Finder to locate genomic regions of interest using the following logic. First, we located all regions containing at least one transposase gene. Within those regions, we next searched for Cas genes located no more than 25 kilobase pairs away from a transposase Transposase-containing regions without at least one nearby Cas gene were discarded from further analy sis. Finally, the remaining regions were further annotated for Tn7 accessory genes (TnsC-TnsE and TniQ), and CRISPR arrays.

We further processed and categorized the Gene Finder hits using Operon Analyzer. To identify Tn7-like CRISPR-transposons, we required each putative operon to contain TnsA, TnsB, TnsC, and at least one Cas gene from Cas5-13; the distance between TnsA, TnsB, and TnsC needed to be less than $500 \mathrm{bp}$; the Cas proteins need to be downstream of TnsA/B/C and the distance between any Cas protein and TnsB needed to be less than $15 \mathrm{kbp}$. We classify this dataset into putative Class 1 sy stems and Class 2 systems based on their Cas signature proteins. Class 1 systems were manually reviewed to confirm the loss of adaptation (Cas1, Cas2) and interference (Cas3) proteins.

To identify non-Tn7 CRISPR-transposons, we required each putative operon to contain a CRISPR array, a transposase, and at least one Cas gene from Cas5-13. We also excluded systems containing Tn7 proteins, Cas 1 , or Cas 2 . We then partitioned this dataset into putative Class 1 systems (defined as loci with any three of Cas5/6/7/8) or Class 2 systems (Cas9, Cas12, or Cas13). For Class 1 systems, we further excluded those containing Cas 3 or Cas 10 . To eliminate systems with fragments of effector proteins or poor matches to unrelated proteins, we required that Cas9 have a size of 2-6 kbp, Cas 12 a size of 3-6 kbp, and Cas 13 a size of 2.5-6 kbp. We eliminated Class 2 systems that were nucleoly tically active (see below), and finally clustered all systems using mmseqs easy-cluster with a minimum sequence ID of 0.95 (59) to simplify manual curation.

\section{BLAST database construction}

To find as many systems as possible, we assembled separate databases for Cas proteins, $\mathrm{Tn} 7$-family proteins, and non- $\mathrm{Tn} 7$ transposases. We also developed databases for common Tn7 attachment sites following a separate effort (21).

We downloaded all available bacterial and archaeal transposase sequences from UniRef50, excluding partial sequences and sequences annotated with the word "zinc" (which tended to be false positives) as well as Tn7-related proteins. All transposases associated with transposons listed in the Transposon Registry (60) were downloaded from NCBI. Finally, 100 transposases associated with each of the major families of insertion sequences were downloaded from NCBI, again excluding partial sequences, and using the 'relevance' sort parameter.

Amino acid sequences for Cas 1-Cas 13 and Tn7 family proteins (TnsATnsE, TniQ) were downloaded from UniRef50 (https://www.uniprot.org/uniref/). Additional Cas12 and Cas13 sequences, representing recently identified variants (e.g. Cas12k), were downloaded from the NCBI protein database (https://www.ncbi.nlm.nih.gov/protein/) and from primary literature sources $(61-63)$.

To eliminate redundant sequences, each database was clustered using CD-HIT (http://weizhongli-lab.org/cd-hit/ (64) with a 50\% sequence identity threshold and $80 \%$ alignment overlap. The clustered datasets were converted to the BLAST database format using makeblastdb (version 2.6.0 of NCBI BLAST+) with the following arguments:

makeblastdb

-in <sequence fasta file>

-title <database name $>$

-out <database name> 
Rybarski, Hu, and Hill et al., 28 July 2021 - preprint copy - BioRxiv

-dbtype prot

-hash_index

The full-length sequences of GuaC (PF00478), RsmJ (PF04378), YciA (PF03061) were downloaded from (http://pfam.xfam.org/). The attachment site SRP-RNA gene (ffs) (RF00169) was downloaded from RFAM (https://rfam.xfam.org/).

To assign putative Cas5-Cas8 proteins to specific Type I CRISPR-Cas subtypes, we manually collected Cas proteins and their assignments from reviews by Koonin and colleagues $(62,65,66)$. All Cas protein sequences were converted into BLAST databases using makeblastdb (version 2.6.0) with default parameters.

\section{De-duplication of putative operons}

Approximately $57 \%$ of the metagenomic systems that passed our initial filter were nearly identical at the nucleotide sequence level. However, exact nucleotide comparisons were too slow to de-duplicate this large dataset. Instead, we considered two systems to be identical if they met the following properties: (1) they had the same protein-coding genes and CRISPR arrays in the same order; (2) the genes had the same relative distances to each other; and (3) the translated sequences of all proteins were identical. This de-duplication was applied to all systems before the downstream analysis.

\section{Self-targeting spacer identification}

Spacer sequences that were identified with PILER-CR were pairwise aligned with the contig sequence that contained them, using the SmithWaterman local alignment function from the parasail library (67), with gap open and gap extension penalties of 8, and using the NUC44 substitution matrix. Spacers with at least $80 \%$ homology to a location in the contig were classified as self-targeting.

For Type V systems, we augmented the CRISPR array search

with minCED 0.4.2 (68) after noticing transposons that were otherwise intact but seemingly lacked CRISPR arrays. The region between Cas 12k and $200 \mathrm{bp}$ after the end of the nearest CRISPR array was used to search for spacers (both atypical and canonical). Targets were searched for in the 500 bp region immediately downstream of the spacer search region, using the method described in the previous paragraph. For Type V systems with multiple Cas $12 \mathrm{k}$ genes, each spacer region was aligned to each target region in order to discover systems where multiple transposons had inserted at the same attachment site.

\section{Phylogenetic analysis}

Alignments of protein sequences were constructed with MAFFT, version v7.310 (69). Phylogenetic analysis was performed on the aligned sequences using the IQ-TREE, version 1.6.12 (70), with automatic model selection. Models used were as follows: Figure 3B: JTT $+F+R 3$, Figure 4B cas6: PMB+G4, Figure 4B cas7: $\mathrm{PMB}+\mathrm{G} 4$, Figure 4C: $\mathrm{PMB}+\mathrm{G} 4$, Supplemental Figure 1 tnsB: LG+R5, Supplemental Figure 1 tnsC: $\mathrm{LG}+\mathrm{G} 4$. Trees were visualized using the Figtree program version 1.4.4.

\section{Classification of nuclease-dead systems}

To identify catalytically inactive Class 2 nucleases, we aligned each nuclease to a reference protein with MAFFT (version v7.310, with the
FFT-NS-2 strategy for Cas9 and Cas12, and FFT-NS-i for Cas13). Cas9 homologs were aligned to SpCas9 (UniProtKB Q99ZW2.1, residues D10 and H840), Cas 12 homologs to AsCas12a (UniProtKB U2UMQ6, residues D908 and E993), and Cas13 homologs to LbuCas13a (UniProtKB C7NBY4.1, residues R472, H477, R1048, and H1053). Mutations of D/E to any thing other than D/E, or H/R to any thing other than $\mathrm{H} / \mathrm{R} / \mathrm{K}$ were considered nuclease dead. As a control, we employed this technique on all 279 Cas $12 \mathrm{k}$ proteins from NCBI as well as LbCas12a and FnCas12a (two known nuclease-active Cas12a proteins) and found that we correctly categorized them all.

\section{Identification of inverted repeats and target site duplications}

To identify inverted repeats, we used Generic Repeat Finder (commit hash: 35b1c4d6b3f6182df02315b98851cd2a30bd6201) (71) with default parameters except as follows:

-c: 0

-s: 15

--min_tr: 15

--min_space <operon length>

--max_space <buffered operon length>

where <operon length> is the length of the putative operon and < buffered operon length $>$ is the length of the putative operon, plus up to $1000 \mathrm{bp}$ to allow a $500 \mathrm{bp}$ buffer on either side of the operon. This detected inverted repeats that were at least $15 \mathrm{bp}$ long. In cases where one inverted repeat fell within the bounds of the putative operon, it was discarded.

\section{Data availability}

All processed data and analysis scripts are available on GitHub at: https://github.com/wilkelab/Metagenomics CAST.

The analysis scripts make use of the gene_finder and operon_analyzer modules of the Opfi package, available on GitHub at: https://github.com/wilkelab/Opfi.

\section{Acknowledgments}

We are grateful to the staff of the Texas Advanced Computing Center for providing computational resources and to members of the Finkelstein and Wilke labs for helpful discussions. This work was supported by NIGMS grants R01GM 124141 (to I.J.F.) \& R01GM088344 (to C.O.W.), the Welch Foundation grant F-1808 (to I.J.F.), and the College of Natural Sciences Catalyst Award for seed funding.

The authors are co-inventors on patent applications filed based on this work.

\section{$\underline{\text { References }}$}

1. J. E. Peters, Targeted transposition with Tn7 elements: safe sites, mobile plasmids, CRISPR/Cas and beyond. Mol. Microbiol. 112, 1635-1644 (2019).

2. J. E. Peters, K. S. Makarova, S. Shmakov, E. V. Koonin, Recruitment of CRISPR-Cas systems by Tn7-like transposons. Proc. Natl. Acad. Sci. 114, E7358-E7366 (2017).

3. T. S. Halpin-Healy, S. E. Klompe, S. H. Sternberg, I. S. Fernández, Structural basis of DNA targeting by a transposon-encoded CRISPR-Cas system. Nature 577, 271-274(2020). 
bioRxiv preprint doi: https://doi.org/10.1101/2021.08.16.456562; this version posted August 17, 2021. The copyright holder for this preprint (which was not certified by peer review) is the author/funder, who has granted bioRxiv a license to display the preprint in perpetuity. It is made available under aCC-BY-NC-ND 4.0 International license.

Rybarski, Hu and Hill et al. 28 July 2021 - preprint copy - BioRxiv

4. N. Jia, W. Xie, M. J. de la Cruz, E. T. Eng, D. J. Patel, Structure-function insights into the initial step of DNA integration by a CRISPR-CasTransposon complex. Cell Res. 30, 182-184(2020).

5. S. E. Klompe, P. L. H. Vo, T.S. Halpin-Healy, S. H. Sternberg, Transposonencoded CRISPR-Cas systems direct RNA-guided DNA integration. Nature 571, 219-225 (2019).

6. B. Wang, W. Xu, H. Yang, Structural basis of a Tn7-like transposase recruitment and DNA loading to CRISPR-Cas surveillance complex. Cell Res. 30, 185-187 (2020).

7. M. Saito, et al., Dual modes of CRISPR-associated transposon homing. Cell (2021) https:/doi.org/10.1016/j.cell.2021.03.006 (April 2,2021).

8. S.-C. Hsieh, J. E. Peters, Tn7-CRISPR-Cas12K elements manage pathway choice using truncated repeat-spacer units to target tRNA attachment sites. bioRxiv, 2021.02.06.429022 (2021).

9. J. Strecker, et al., RNA-guided DNA insertion with CRISPR-associated transposases. Science 365, 48-53(2019).

10. K. S. Makarova, et al., Evolutionary classification of CRISPR-Cas sy stems: a burst of class 2 and derived variants. Nat. Rev. Microbiol. 18, 67-83 (2020).

11. C. Camacho, et al., BLAST+: architecture and applications. BMC Bioinformatics 10,421 (2009).

12. R. C. Edgar, PILER-CR: fast and accurate identification of CRISPR repeats. BMC Bioinformatics 8, 18 (2007).

13. V. Burrus, G. Pavlovic, B. Decaris, G. Guédon, Conjugative transposons: the tip of the iceberg. Mol. Microbiol. 46, 601-610(2002).

14. A. Mark Osborn, D. Böltner, When phage, plasmids, and transposons collide: genomic islands, and conjugative- and mobilizable-transposons as a mosaic continuum. Plasmid 48, 202-212(2002).

15. G. Faure, et al., CRISPR-Cas in mobile genetic elements: counter-defence and beyond. Nat. Rev. Microbiol. 17,513-525(2019).

16. NCBI Resource Coordinators, Database resources of the NationalCenter for Biotechnology Information. Nucleic Acids Res. 46, D8-D13 (2018).

17. A. L. Mitchell, et al., MGnify: the microbiome analysis resource in 2020. Nucleic Acids Res. 48, D570-D578 (2020).

18. The UniProt Consortium, UniProt: a worldwide hub of protein knowledge. Nucleic Acids Res. 47, D506-D515 (2019).

19. E. V. Koonin, K. S. Makarova, Y. I. Wolf, M. Krupovic, Evolutionary entanglement of mobile genetic elements and host defence sy stems: guns for hire. Nat. Rev. Genet. 21, 119-131 (2020).

20. A. R. Parks, J. E. Peters, Tn7 elements: Engendering diversity from chromosomes to episomes. Plasmid 61, 1-14(2009).

21. M. T. Petassi, S.-C. Hsieh, J. E. Peters, Guide RNA Categorization Enables Target Site Choice in Tn7-CRISPR-Cas Transposons. Cell 183, 17571771.e18(2020).

22. Z. Li, H. Zhang, R. Xiao, L. Chang, Cryo-EM structure of a type I-F CRISPR RNA guided surveillance complex bound to transposition protein TniQ. Cell Res. 30, 179-181 (2020).

23. M. F. Rollins, J. T. Schuman, K. Paulus, H. S. T. Bukhari, B. Wiedenheft, Mechanism of foreign DNA recognition by a CRISPR RNA-guided surveillance complex from Pseudomonas aeruginosa. Nucleic Acids Res. $\mathbf{4 3}$, 2216-2222(2015).

24. K. Y. Choi, Y. Li, R. Sarnovsky, N. L. Craig, Direct interaction bet ween the TnsA and TnsB subunits controls the heteromeric Tn 7 transposase. Proc. Natl. Acad. Sci. U. S.A. 110, E2038-E2045(2013).

25. R. Pinilla-Redondo, et al., TypeIVCRISPR-Cas systems are highly diverse and involved in competition between plasmids. Nucleic Acids Res. 48, 20002012 (2020).

26. H. N. Taylor, et al., Positioning Diverse Type IV Structures and Functions Within Class 1 CRISPR-Cas Systems. Front. Microbiol. 12 (2021).

27. J. Strecker, A. Ladha, K. S. Makarova, E. V. Koonin, F. Zhang, Response to Comment on "RNA-guided DNA insertion with CRISPR-associated transposases." Science 368 (2020).
28. K. Y. Choi, J. M. Spencer, N. L. Craig, The $\operatorname{Tn} 7 \operatorname{transposition~regulator~TnsC~}$ interacts with the transposase subunit TnsB and target select or TnsD. Proc. Natl. Acad. Sci. 111, E2858-E2865 (2014).

29. J.-U. Park, et al., Structural basis for target-site selection in RNA-guided DNA transposition systems. bioRxiv, 2021.05.25.445634(2021).

30. J. E. Peters, “Tn7” in Mobile DNA III, (John Wiley \& Sons, Ltd, 2015), pp. 647-667.

31. S. Ae, C. Nl, Avoiding Self: Two Tn7-encoded Proteins Mediate Target Immunity in Tn7 Transposition. EMBO J. 16 (1997) Available at: https://pubmed.ncbi.nlm.nih.gov/9362496/ [Accessed May 19, 2020].

32. Z. Skelding, J. Queen-Baker, N. L. Craig, Alternative interactions bet ween the $\mathrm{Tn} 7 \mathrm{transposase}$ and the $\mathrm{Tn} 7$ target DNA binding protein regulate target immunity and transposition. EMBOJ. 22,5904-5917 (2003).

33. A. W. Kingston, C. Ponkratz, E. A. Raleigh, Rpn (YhgA-Like) Proteins of Escherichia coli K-12 and Their Contribution to RecA-Independent Horizontal Transfer. J. Bacteriol. 199 (2017).

34. A. K. Aggarwal, Structure and function of restriction endonucleases. Curr. Opin. Struct. Biol. 5, 11-19(1995).

35. A. B. Hickman, et al., Unexpected structural diversity in DNA recombination: the restriction endonuclease connection. Mol. Cell 5, 10251034 (2000).

36. K. Steczkiewicz, A. Muszewska, L. Knizewski, L. Rychlewski, K. Ginalski, Sequence, structure and functional diversity of PD-(D/E)XK phosphodiesterase superfamily. Nucleic Acids Res. 40, 7016-7045 (2012).

37. R. Missich, et al., The replisome organizer (G38P) of Bacillus subtilis bacteriophage SPP1 forms specialized nucleoprotein complexes with two discrete dist ant regions of the SPP1 genome. J. Mol. Biol. 270, 50-64 (1997).

38. T. Jacobsen, et al., Characterization of Cas12a nucleases reveals diverse PAM profiles between closely-related orthologs. Nucleic Acids Res. 48, 5624-5638(2020).

39. R. T. Leenay, et al., Identifying and Visualizing Functional PAM Diversity across CRISPR-Cas Systems. Mol. Cell 62, 137-147 (2016).

40. T. Yamano, et al., Crystal Structure of Cpf1 in Complex with Guide RNA and T arget DNA. Cell 165,949-962 (2016).

41. I. Fonfara, H. Richter, M. Bratovič, A. Le Rhun, E. Charpentier, The CRISPR-associated DNA-cleaving enzyme Cpf1 also processes precursor CRISPR RNA. Nature 532, 517-521 (2016).

42. Y. Jeon, et al., Direct observation of DNA target searching and cleavage by CRISPR-Cas12a. Nat. Commun. 9, 2777 (2018).

43. D. Singh, et al., Real-time observation of DNA target interrogation and product release by the RNA-guided endonuclease CRISPR Cpf1 (Cas12a). Proc. Natl. Acad. Sci. 115, 5444-5449 (2018).

44. I. Strohkendl, F. A. Saifuddin, J. R. Rybarski, I. J. Finkelstein, R. Russell, Kinetic Basis for DNA Target Specificity of CRISPR-Cas12a.Mol. Cell 71, 816-824.e3(2018).

45. D. C. Swarts, M. Jinek, Mechanistic Insights into the cis- and trans-Acting DNase Activities of Cas12a. Mol. Cell 73, 589-600.e4 (2018).

46. E. V. K. Sergey A. Shmakov, Systematic prediction of functionally linked genes in bacterial and archaeal genomes. Nat. Protoc. (2019).

47. S. A. Shmakov, K. S. Makarova, Y. I. Wolf, K. V. Severinov, E. V. Koonin, Systematic prediction of genes functionally linked to CRISPR-Cas systems by gene neighborhood analysis. Proc. Natl. Acad. Sci. 115, E5307-E5316 (2018).

48. D. Gleditzsch, et al., Modulating the Cascade architecture of a minimal Type I-F CRISPR-Cas system. Nucleic Acids Res. 44,5872-5882 (2016).

49. K. Kuznedelov, et al., Altered stoichiometry Escherichia coli Cascade complexes with shortened CRISPR RNA spacers are capable of interference and primed adaptation. Nucleic Acids Res. 44, 10849-10861 (2016).

50. I. Songailiene, et al., Decision-Making in Cascade Complexes Harboring crRNAs of Altered Length. Cell Rep. 28, 3157-3166.e4 (2019).

51. D. Tuminauskaite, et al., DNA interference is controlled by R-loop length in a type I-F1 CRISPR-Cas system. BMC Biol. 18, 65 (2020). 
bioRxiv preprint doi: https://doi.org/10.1101/2021.08.16.456562; this version posted August 17, 2021. The copyright holder for this preprint (which was not certified by peer review) is the author/funder, who has granted bioRxiv a license to display the preprint in perpetuity. It is made available under aCC-BY-NC-ND 4.0 International license.

Rybarski, Hu, and Hill et al., 28 July 2021 - preprint copy - BioRxiv

52. V. Hoikkala, Cooperation between Different CRISPR-Cas Types Enables Adaptation in an RNA-Targeting System. $m$ Bio (2021) (June 18,2021).

53. J. N. Vink, J. H. Baijens, S. J. Brouns, Comprehensive PAM prediction for CRISPR-Cas systems reveals evidence for spacer sharing, preferred strand targeting and conserved links with CRISPR repeats. bioRxiv, 2021.05.04.442622 (2021).

54. G. Amitai, R. Sorek, CRISPR-Cas adaptation: insights into the mechanism of action. Nat. Rev. Microbiol. 14,67-76 (2016).

55. B. D. Lavoie, G. Chaconas, Site-specific HU binding in the Mu transpososome: conversion of a sequence-independent DNA-binding protein into a chemical nuclease. Genes Dev. 7, 2510-2519(1993).

56. FastQC, FastQC: A quality control tool for high throughput sequence data (2015).

57. N. A. Joshi, J. N. Fass, Sickle: A sliding-window, adaptive, quality-based trimming tool for FastQ files (Version 1.33) [Software] (2011)

58. D. Li, C.-M. Liu, R. Luo, K. Sadakane, T.-W. Lam, MEGAHIT: an ultrafast single-node solution for large and complex metagenomics assembly via succinct de Bruijn graph. Bioinforma. Oxf. Engl. 31, 1674-1676(2015).

59. M. Steinegger, J. Söding, MMseqs2 enables sensitive protein sequence searching for the analysis of massive data sets. Nat. Biotechnol. 35, 10261028 (2017).

60. S. Tansirichaiya, Md. A. Rahman, A. P. Roberts, The Transposon Registry. Mob. DNA 10, 40 (2019).
61. P. Pausch, et al., CRISPR-Cas $\Phi$ from huge phages is a hypercompact genome editor. Science 369, 333-337 (2020).

62. S. Shmakov, et al., Diversity and evolution of class 2 CRISPR-Cas systems. Nat. Rev. Microbiol. 15,169-182(2017).

63. W. X. Yan, et al., Functionally diverse type VCRISPR-Cas systems. Science 363, 88-91 (2019).

64. L. Fu, B. Niu, Z. Zhu, S. Wu, W. Li, CD-HIT: accelerated for clustering the next-generation sequencing dat a. Bioinformatics 28, 3150-3152 (2012).

65. D. Burstein, et al., New CRISPR-Cas systems from uncultivated microbes. Nature 542, 237-241 (2017).

66. K. S. Makarova, et al., An updated evolutionary classification of CRISPRCas systems. Nat. Rev. Microbiol. 13,722-736(2015).

67. J. Daily, Parasail: SIMD C library for global, semi-global, and local pairwise sequence alignments. BMC Bioinformatics 17, 81 (2016).

68. C. Skennerton, MinCED (2019).

69. K. Katoh, D. M. Standley, MAFFT Multiple Sequence Alignment Software Version 7: Improvements in Performance and Usability. Mol. Biol. Evol. 30, $772-780$ (2013).

70. L.-T. Nguyen, H. A. Schmidt, A. von Haeseler, B. Q. Minh, IQ-TREE: A Fast and Effective Stochastic Algorithm for Estimating MaximumLikelihood Phylogenies. Mol. Biol. Evol. 32, 268-274 (2015).

71. J. Shi, C. Liang, Generic Repeat Finder: A High-Sensitivity Tool for Genome-Wide De Novo Repeat Detection1. Plant Physiol. 180, 1803-1815 (2019). 